\title{
Characterization of a Novel Mitovirus Infecting Melanconiella theae Isolated From Tea Plants
}

\author{
Karim Shafik ${ }^{1,2,3,4,5 t}$, Muhammad Umer ${ }^{1,3,4,5 t}$, Huafeng You ${ }^{1,3,4,5}$, \\ Hamdy Aboushedida ${ }^{1,2,3,4,5}$, Zhenhua Wang ${ }^{6}$, Dejiang $\mathrm{Ni}^{3}$ and Wenxing $\mathrm{Xu}^{1,3,4,5 *}$ \\ ${ }^{1}$ Hubei Hongshan Laboratory, Wuhan, China, ${ }^{2}$ Department of Plant Pathology, Faculty of Agriculture, Alexandria University, \\ Alexandria, Egypt, ${ }^{3}$ Key Laboratory of Horticultural Plant Biology, College of Horticulture and Forestry Sciences, Ministry \\ of Education, Huazhong Agricultural University, Wuhan, China, ${ }^{4}$ Key Lab of Plant Pathology of Hubei Province, Wuhan, \\ China, ${ }^{5}$ College of Plant Science and Technology, Huazhong Agricultural University, Wuhan, China, ${ }^{6}$ Technology Center of \\ Wuhan Customs District, Wuhan, China
}

A dsRNA segment was identified in the fungus Melanconiella theae isolated from tea plants. The complete dsRNA sequence, determined by random cloning together with RACE protocol, is 2,461 bp in length with an AU-rich content (62.37\%) and comprises a single ORF of 2,265-nucleotides encoding an RNA-dependent RNA-polymerase (RdRp, 754 amino acids in size). The terminus sequences can fold into predicted stable stemloop structures. A BLASTX and phylogenetic analysis revealed the dsRNA genome shows similarities with the RdRp sequences of mitoviruses, with the highest identity of $48 \%$ with those of grapevine-associated mitovirus 20 and Colletotrichum fructicola mitovirus 1. Our results reveal a novel member, tentatively named Melanconiella theae mitovirus 1 (MtMV1), belongs to the family Mitoviridae. MtMV1 is capsidless as examined by transmission electron microscope, efficiently transmitted through conidia as 100 conidium-generated colonies were analyzed, and easily eliminated by hyphal tipping method combined with green-leaf tea powder. MtMV1 has a genomic sequence obviously divergent from those of most members in the family Mitoviridae and some unique characteristics unreported in known members. This is the first report of a mycovirus infecting Melanconiella fungi to date.

Keywords: mycovirus, mitovirus, mitochondrial virus, MtMV1, Melanconiella theae, Camellia sinensis

\section{INTRODUCTION}

Mycoviruses (also known as fungal viruses) are widespread in all major taxa of fungi (Strauss et al., 2000; Lim et al., 2005; Ro et al., 2007; Stielow et al., 2011; Magae, 2012). Mycoviruses are usually associated with symptomless (cryptic or latent symptoms) infections (Kamaruzzaman et al., 2019). However, some can attenuate the virulence of their fungal hosts and contribute as biocontrol agents for preventing the diseases induced by phytopathogenic fungi (Wu et al., 2007; Ghabrial and Suzuki, 2009; Pearson et al., 2009; Abid et al., 2018; Mizutani et al., 2018). According to the International Committee on Taxonomy of Virus ${ }^{1}$, mycoviruses have been taxonomically grouped into 19 families, including six with dsRNA genomes (Chrysoviridae, Partitiviridae,

\footnotetext{
${ }^{1}$ https://talk.ictvonline.org/taxonomy/
} 
Quadriviridae, Reoviridae, Totiviridae, and Megabirnaviridae), nine with positive-sense single-stranded RNA (+ ssRNA) (Alphaflexiviridae, Barnaviridae, Botourmiaviridae, Deltafleiviridae, Endornaviridae Gammaflexiviridae, Hypoviridae, Mitoviridae, and Narnaviridae) (Kamaruzzaman et al., 2019), one with negative-sense ssRNA (-ssRNA; Mymonaviridae) (Liu et al., 2014), one with ssDNA (Genomoviridae) (Yu et al., 2010), two with reverse-transcribing RNA (Metaviridae and Pseudoviridae), and unclassified taxa (Liu et al., 2014; He et al., 2018). The number of mycovirus primarily increased in recent years through deep sequencing analysis, and most of the mycoviruses are derived from major phytopathogenic fungi isolated from crops, fruits, and forest trees. In contrast, to date, there are much less reports on mycoviruses associated with fungi isolated from tea plants [Camellia sinensis (L.) O. Kuntze], which originated from China and has been cultivated for over 3,000 years.

Mitoviruses (family Mitoviridae) represent the simplest group of all RNA viruses (Hillman and Cai, 2013), with a unique genome of + ssRNA which ranges in size between 2.0 and $4.5 \mathrm{~kb}$, encompassing a single long open reading frame (ORF) which encodes a putative RNA-dependent RNA polymerase (RdRp) with six conserved amino acid motifs (I-IV) (Osaki et al., 2015; Ran et al., 2016; Chen et al., 2017; Wang et al., 2019; Kocanová et al., 2020; Liu et al., 2021). Their Genomes are AU rich, especially in the third position of codons (Hong et al., 1998; Xie and Ghabrial, 2012; Liu et al., 2019; Torres-Trenas and Pérez-Artés, 2020; Wang et al., 2021). Mitoviral genomes are characterized by multiple UGA codons encode tryptophan (Trp) instead of stop translation (Nibert, 2017; Nibert et al., 2019); however, the termination codon is usually UAA and sometimes UAG (Nibert, 2017). The terminus sequences could be folded into stable genomic secondary structures that have crucial roles in replicating and protecting their genome (Khalifa and Pearson, 2013; Sahin and Akata, 2019; Torres-Trenas and Pérez-Artés, 2020; Liu et al., 2021). However, mitoviruses are initially found infecting fungi, they have recently been reported from plants as expected infectious entities (Nibert et al., 2018; Nerva et al., 2019; Vong et al., 2019). They are transmitted through horizontal transmission (hyphal anastomosis and heterokaryosis) or vertical transmission (spores) (Romeralo et al., 2018; Kamaruzzaman et al., 2019). Mitoviruses are the only viruses associated with the mitochondria in their hosts (Cole et al., 2000; Osaki et al., 2005; Nibert, 2017; Fonseca et al., 2021) and often led to hypovirulence in their fungal hosts (Nuss, 2005; Xu et al., 2015; Yang et al., 2018).

The genus Melanconiella (order Diaporthales, ascomycetes) involves saprophytes and endophytes as well as phytopathogenic fungi causing cankers, diebacks, and rots on crops, ornamentals, and forest trees (Du et al., 2017). Members of the fungus Melanconiella were mostly observed to be highly host-specific pathogenic and endophytic fungi of the host family Betulaceae especially in the north temperate zone (Voglmayr et al., 2012). To our knowledge, no mycoviruses have been reported from Melanconiella theae. Here, we characterized a novel mitovirus from $M$. theae that represent the first mycovirus from Melanconiella fungi. It will expand our understanding of the origin, ecology, and evolutionary pathways of mycoviruses (Ghabrial, 1998; Ghabrial and Suzuki, 2009).

\section{MATERIALS AND METHODS}

\section{Fungus Isolation and Identification}

Melanconiella theae strains WJB-5 (Supplementary Figure 1), WJB-1-1, WJB-1-2, WJB-1-9, WJB-1-12, WJB-1-20, WJT-1-1, WJT-1-3, WJT-1-7, WJT-1-9, WJT-1-12, and WJT-1-13 were isolated from naturally infected tea plants showing necrotic symptoms (Supplementary Figure 1A) collected from Xuanen county, Hubei province, China, and identified based on the internal transcribed spacer (ITS) region. Of these strains, WJB-19 and WJB-1-12 have the same genetic background with WJT-1-9 and WJT-1-12, respectively, since they were derived from the same strain by culturing single mycelium. Fungal mycelia of these strains were grown on PDA for a week at $25^{\circ} \mathrm{C}$ and then stored in a $25 \%(\mathrm{v} / \mathrm{v})$ glycerol solution at $-70^{\circ} \mathrm{C}$ until use.

\section{Double-Stranded RNA (dsRNA) Extraction}

Viral dsRNA was extracted from the fungal mycelial mass using the silica spin column-based method as described previously (Yang et al., 2017). The dsRNAs extracted from $M$. theae strains WJB-1-1, WJB-1-2, WJB-1-9, WJB-1-12, WJB-1-20, and WJB-5, and those from strain WJB-5 were used for viral genome characterization and treated with RNase-free DNase I and S1 Nuclease to remove any DNA and ssRNA remains, fractionated by agarose gel $(1 \%, \mathrm{w} / \mathrm{v})$ electrophoresis, and detected by UV transillumination after staining with ethidium bromide $(0.1 \mathrm{mg} / \mathrm{mL})$.

\section{Complementary DNA (cDNA) Cloning and Sequencing, and RT-PCR Amplication}

Purified dsRNA from WJB-5 was used as a template for cDNA synthesis following the previously described method (Xie et al., 2011) using a cDNA synthesis kit (Promega, Madison, WI, United States) and tagged random dN6 primer, 05RACE-3RT (Supplementary Table 1). The resulting cDNA was PCR-amplified using $2 \times$ Gimmico GimiTaq PCR Mix (Gimmico Biotech Co., Wuhan, China) and 05RACE-3 primer (Supplementary Table 1). RT-PCR products were purified and then ligated to the pMD18-T vector (TaKaRa, Beijing, China), then transformed into competent cells of Escherichia coli $\mathrm{DH} 5 \alpha$. Positive clones with cDNA inserts were selected on Luria-Bertani (LB) agar medium containing ampicillin $(50 \mu \mathrm{g} / \mathrm{mL})$, checked with PCR using M13F-47/M13R-48 primers (Supplementary Table 1), and then submitted for sequencing. Specific primers, MtMV1-F and MtMV1-R (Supplementary Table 1) were designed for rapid amplification of cDNA ends (RACE) to amplify terminal sequences of the genome that were not cloned by the initial random cDNA synthesis. For RACE, the PC3-T7 loop adapter (Supplementary Table 1) was ligated to the $3^{\prime}$ end of each strand in the purified dsRNA using T4 RNA ligase (TaKaRa, 

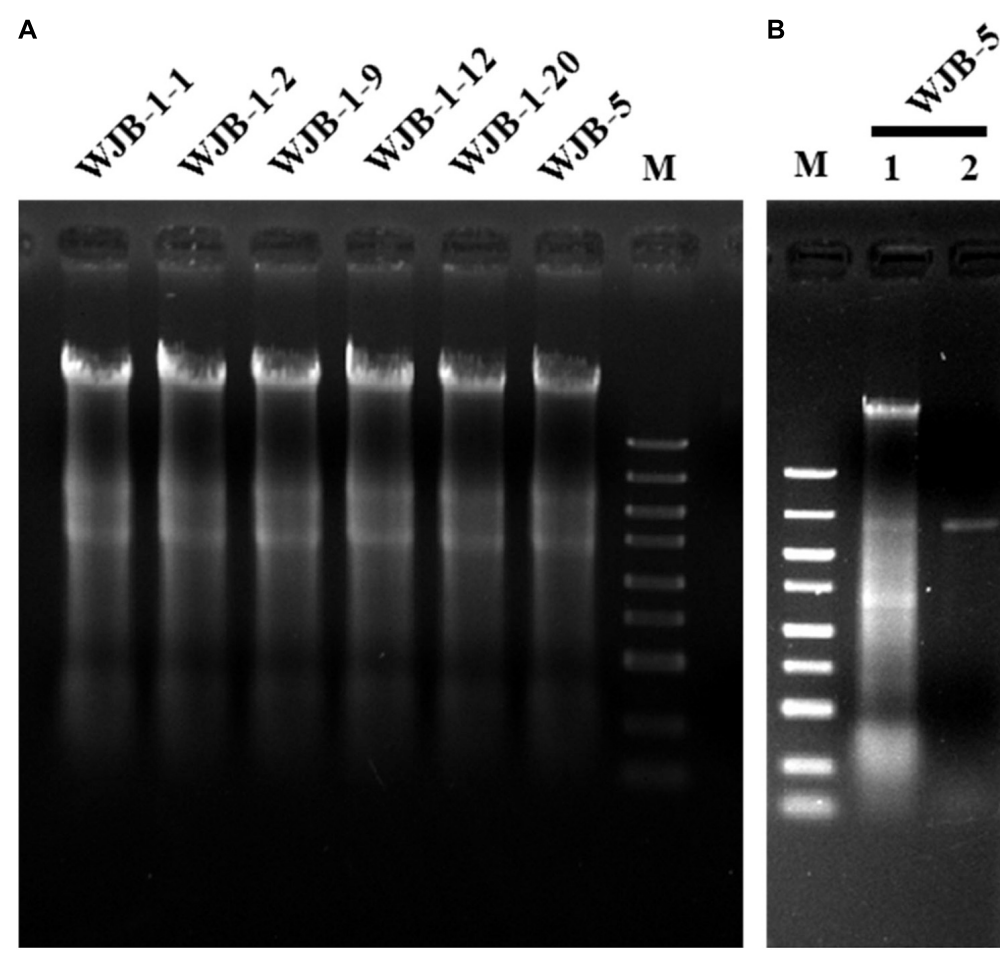

C
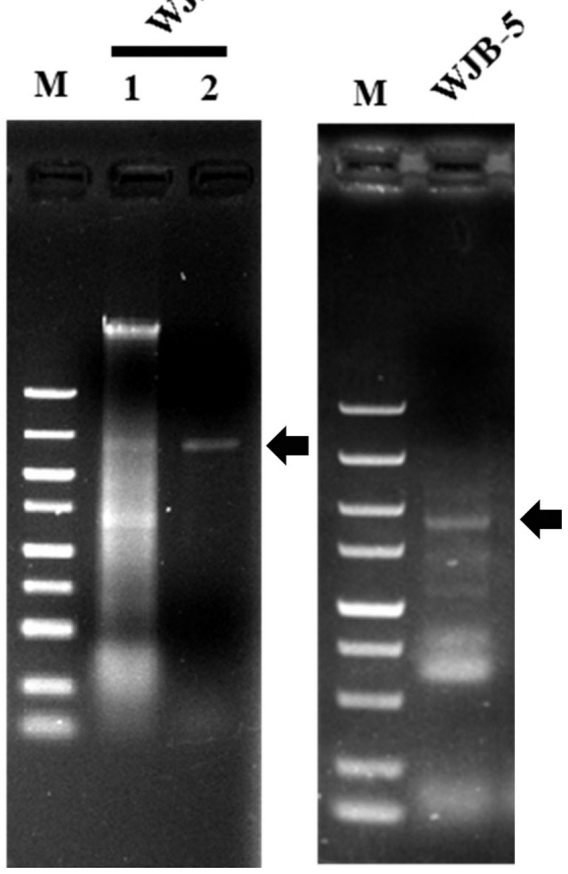

FIGURE 1 | Agarose gel electrophoresis of nucleic acids extracted from Melanconiella theae strains, enzyme digest and RT-PCR identification of MtMV1. (A) Nucleic acids extracted from strains WJB-1-1, WJB-1-2, WJB-1-9, WJB-1-12, WJB-1-20, and WJB-5. (B) The nucleic acids extracted from strain WJB-5 were untreated (Lane 1) or treated with S1 nuclease (Lane 2). (C) RT-PCR identification of MtMV1 in strain WJB-5. M: DL5000 DNA marker (100; 250; 500; 750; 1,000; 1,500; 2,000; 3,000; and 5,000 bp). The arrows indicate the target bands.

Beijing, China) at $16^{\circ} \mathrm{C}$ for $18 \mathrm{~h}$, as described previously (Ran et al., 2016). The oligonucleotide-ligated dsRNA was purified, denatured in DMSO, and subsequently reverse transcribed. RACE and nested PCR amplification were performed using sets of MtMV1-F/PC2 and PC2/MtMV1-R primers (Supplementary Table 1) to amplify the $3^{\prime}$ - and $5^{\prime}$-termini respectively. PCR amplicons were purified, cloned into a pMD18-T vector, and sequenced. All amplicons were sequenced by Tsingke Biological Technology Co., LTD., Wuhan, China. Sequence homology searches were performed with the BLASTX search tool on the National Center for Biotechnology Information, $\mathrm{NCBI}^{2}$. Sequence assemblies from the cDNA and the RACE clones were manipulated using DNAMAN software (version 7.0.2; Lynnon Corp., Vaudreuil-Dorion, QC, Canada) to obtain the complete genome sequence of the dsRNA extracted from the strain WJB-5.

RT-PCR amplification was performed using a specific primer pair (MtMV1-F1: 5'-GTCTTATCCGTGTATTCTGGG-3'; MtMV1-R1: 5'-GGCTTGAGGAACATTGAGA-3'), generating a 1981-bp fragment. An annealing temperature of $56^{\circ} \mathrm{C}$ was used with a PCR Thermal Cycler (Model A200, LongGene, China).

\section{Phylogenetic and Sequence Analyses}

The viral ORF was identified using Expasy ${ }^{3}$ website, and the amino acid (aa) sequences encoded by the ORF were deduced.

\footnotetext{
${ }^{2}$ http://www.ncbi.nlm.nih.gov/
}

${ }^{3}$ https://web.expasy.org/translate/
The yeast mitochondrial genetic code was used for the translation of the ORF. The mycovirus sequence was searched using the NCBI BLASTX program to identify similar mycoviruses for phylogenetic analysis. Maximum-likelihood (ML) phylogenetic tree with 1,000 bootstrap replicates was constructed using DNAMAN software (version 7.0.2; Lynnon Corp., VaudreuilDorion, QC, Canada) for the amino acid sequences of RdRp from WJB-5 dsRNA along with 26 mitoviruses and 4 narnaviruses retrieved from the GenBank database. The evolutionary distances were computed using the Poisson correction method (uniform rates between sites). The potential RNA secondary structures of the $5^{\prime}$ - and $3^{\prime}$ - terminal sequences were predicted, and the free energy $(\Delta G)$ was estimated using CLC genomics workbench software (version 21.0.4; QIAGEN Aarhus, Aarhus, Denmark) as described previously (Liu and Di, 2020). Mitoviral RdRp characteristic motifs were searched using the conserved domain database (CDD) search ${ }^{4}$ provided by NCBI, and multiple amino acid alignments.

\section{Association of Strain WJB-5 With Viral-Like Particles}

Mycelium plugs of the strain WJB-5 were grown on an autoclaved cellophane membrane placed on media for 8 days (d) at $25^{\circ} \mathrm{C}$. Minor modifications were adopted to isolate and purify viral

${ }^{4}$ https://www.ncbi.nlm.nih.gov/cdd 


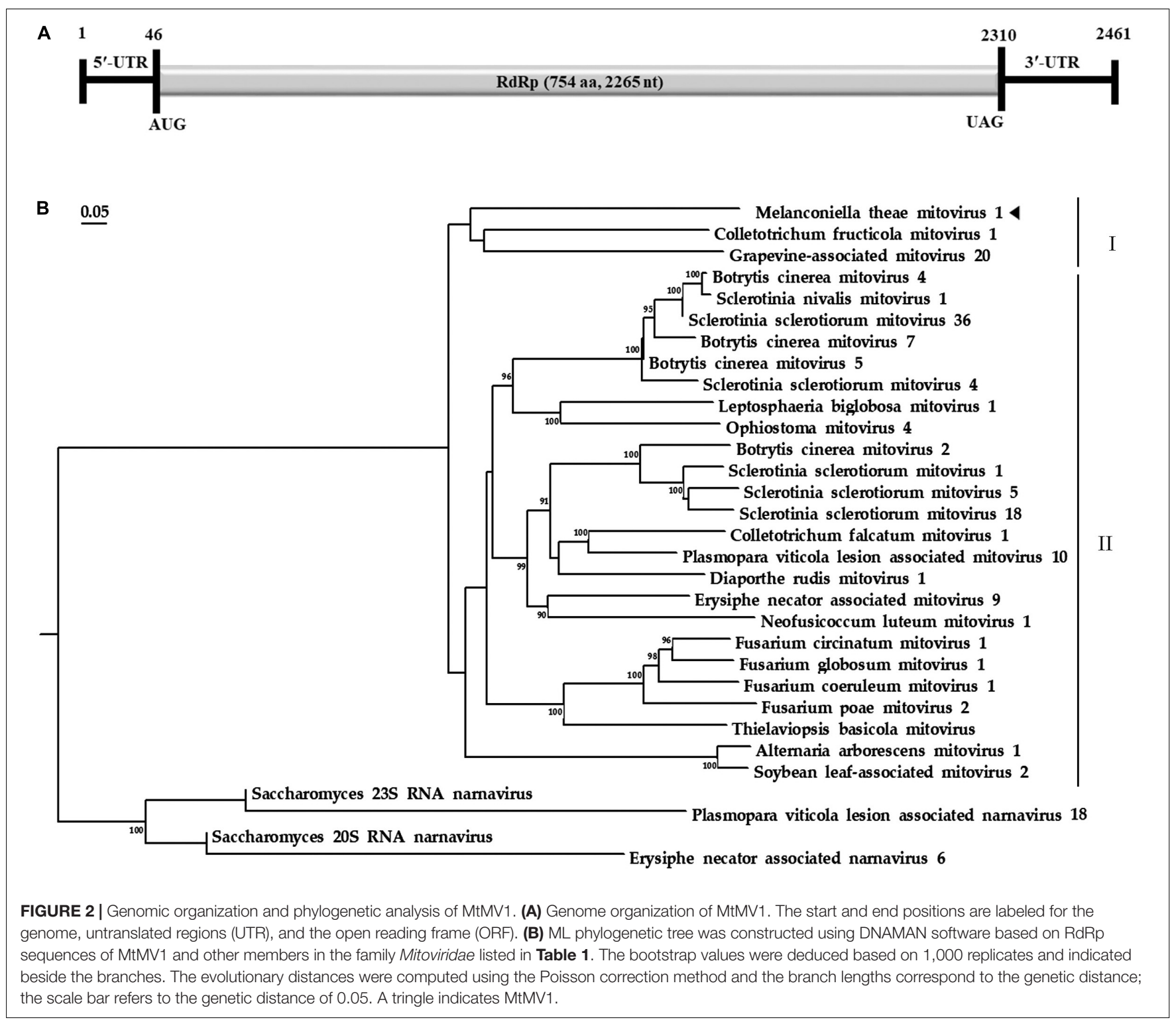

particles in the previously described method (Ran et al., 2016). In brief, $35 \mathrm{~g}$ mycelia of WJB-5 strain were harvested and crushed in the presence of liquid nitrogen. After that, the crushed mycelia were transferred into new sterile tubes containing 3 volumes of cold phosphate buffer $(0.1 \mathrm{M}$ sodium phosphate, $\mathrm{pH}$ 7.0, containing $0.2 \mathrm{M} \mathrm{KCl}$ and $0.5 \% \beta$-mercaptoethanol) and gently mixed. The homogenate was centrifuged at $10,000 \mathrm{rpm}$ and $4^{\circ} \mathrm{C}$ for $15 \mathrm{~min}$ to remove the hyphal debris. After three ultracentrifugation cycles, virus particles were purified using a stepwise sucrose gradient of $20-50 \%$ (W/V). Following sucrose gradient ultracentrifugation, sucrose fractions were collected, diluted, and subjected to ultracentrifugation to pellet virus particles, and the pellet was resuspended again in $150 \mu \mathrm{L}$ of phosphate buffer $(0.1 \mathrm{~mol} / \mathrm{L}$ sodium phosphate, $\mathrm{pH} 7.0)$. Viral dsRNA was recovered from purified viral particles by a phenolchloroform-based technique as described previously (Toni et al., 2018), fractionated by agarose gel (1\%,w/v) electrophoresis and detected by UV transillumination. For negative staining, $10 \mu \mathrm{L}$ purified suspension of the virus was loaded on a hydrophobic parafilm surface, and subsequently copper grids with a carbonformvar coating (200-mesh) were floated onto them for $3 \mathrm{~min}$. After drying with a filter paper, the grids plated with virus particles were immediately refloated for $3 \mathrm{~min}$ in a drop of $2 \%$ (W/V) phosphotungstic acid solution. The excess suspension was removed using filter paper, and the grids were then air-dried for a few min. Finally, transmission electron microscopy (TEM) was used to visualize the samples.

\section{Attempts to Cure Melanconiella Strains}

To eliminate the mycovirus from WJB-5 and five other (WJB1-1, WJB-1-2, WJB-1-9, WJB-1-12, and WJB-1-20) strains of Melanconiella theae, hyphal tipping method together with thermal/dark treatment were conducted. All strains were separately incubated on PDA-T [potato dextrose agar medium 
TABLE 1 | Comparison of genomic information of MtMV1 and other mitoviruses.

\begin{tabular}{|c|c|c|c|c|c|c|c|}
\hline Mitoviruses & Genome (nt) & RdRp (aa) & Tryp codons & Stop codon & 5t-UTR size & 3'-UTR size & Accession no. \\
\hline Melanconiella theae mitovirus 1 & 2,461 & 754 & 12 & UAG & 45 & 151 & MW802251 \\
\hline Alternaria arborescens mitovirus 1 & 2,506 & 717 & 10 & UAG & 218 & 134 & NC_030747.1 \\
\hline Botrytis cinerea mitovirus 2 & 2,496 & 710 & 11 & UAA & 325 & 40 & LN827945.1 \\
\hline Botrytis cinerea mitovirus 4 & 2,768 & 731 & 19 & UAA & 472 & 100 & NC_028474.1 \\
\hline Botrytis cinerea mitovirus 5 & 2,721 & 731 & 19 & UAA & 452 & 73 & MN617167.1 \\
\hline Botrytis cinerea mitovirus 7 & 2,705 & 731 & 19 & UAA & 461 & 48 & MN617168.1 \\
\hline Colletotrichum falcatum mitovirus 1 & 2,283 & 703 & 12 & UAA & 136 & 35 & MK279482.1 \\
\hline Colletotrichum fructicola mitovirus 1 & 2,398 & 709 & 10 & UAA & 216 & 51 & LC497424.1 \\
\hline Diaporthe rudis mitovirus 1 & 2,455 & 701 & 8 & UAA & 302 & 47 & MT216310.1 \\
\hline Erysiphe necator associated mitovirus 9 & 2,453 & 717 & 11 & UAG & 232 & 67 & MN557015.1 \\
\hline Fusarium circinatum mitovirus 1 & 2,419 & 731 & 13 & UAG & 156 & 67 & KF803546.1 \\
\hline Fusarium coeruleum mitovirus 1 & 2,423 & 757 & 16 & UAG & 79 & 70 & NC_026622.1 \\
\hline Fusarium globosum mitovirus 1 & 2,414 & 717 & 15 & UAG & 188 & 72 & NC_026621.1 \\
\hline Fusarium poae mitovirus 2 & 2,414 & 764 & 13 & UAG & 65 & 54 & NC_030862.1 \\
\hline Grapevine-associated mitovirus 20 & 2,467 & 792 & 15 & UAG & 49 & 39 & MW648468.1 \\
\hline Leptosphaeria biglobosa mitovirus 1 & 2,568 & 756 & 13 & UAA & 240 & 56 & NC_040819.1 \\
\hline Neofusicoccum luteum mitovirus 1 & 2,389 & 710 & 9 & UAA & 128 & 127 & NC_035114.1 \\
\hline Ophiostoma mitovirus 4 & 2,599 & 783 & 13 & UAA & 204 & 43 & NC_004052.1 \\
\hline Plasmopara viticola lesion associated mitovirus 10 & 2,303 & 705 & 10 & UAA & 182 & $2^{*}$ & MN539771.1 \\
\hline Sclerotinia nivalis mitovirus 1 & 2,720 & 731 & 19 & UAA & 467 & 57 & KT365895.1 \\
\hline Sclerotinia sclerotiorum mitovirus 1 & 2,513 & 691 & 12 & UAG & 418 & 19 & JQ013377.1 \\
\hline Sclerotinia sclerotiorum mitovirus 4 & 2,744 & 731 & 19 & UAA & 464 & 84 & JX401538.1 \\
\hline Sclerotinia sclerotiorum mitovirus 5 & 2,497 & 718 & 10 & UAG & 291 & 49 & KJ462511.1 \\
\hline Sclerotinia sclerotiorum mitovirus 18 & 2,509 & 722 & 10 & UAG & 299 & 41 & KP900925.1 \\
\hline Sclerotinia sclerotiorum mitovirus 36 & 2,732 & 739 & 19 & UAA & 432 & $80^{\star}$ & MT646380.1 \\
\hline Soybean leaf-associated mitovirus 2 & 2,477 & 721 & 10 & UAG & 190 & 121 & KT598239.1 \\
\hline Thielaviopsis basicola mitovirus & 2,896 & 705 & 12 & UAG & 427 & 351 & DQ173015.2 \\
\hline
\end{tabular}

*Probably incomplete genome.

containing $0.4 \%(\mathrm{w} / \mathrm{v})$ green-leaf tea powder] (Yang and Zhang, 2019) media in the dark at $20^{\circ} \mathrm{C}$ for 3 days. The hyphal tips in the colony margin were individually cut using a fine needle, transferred again to PDA-T in new Petri dishes (1 hyphal tip per dish), and incubated simultaneously. The colonies were subcultured on PDA-T at $20^{\circ} \mathrm{C}$ in dark conditions for three consecutive subculturing. Finally, the subculturing was achieved on PDA through incubation at $20^{\circ} \mathrm{C}$ and then used to detect MtMV1 dsRNA. To emphasize that the $M$. theae strains got cured from MtMV1, total RNA was extracted and RT-PCR amplification was performed using specific primers (MtMV1F1: $\quad 5^{\prime}$-GTCTTATCCGTGTATTCTGGG-3'; MtMV1-R1: $5^{\prime}$-GGCTTGAGGAACATTGAGA-3'), as previously described.

\section{Analysis of Mycovirus Transmission Through Conidia}

Conidia of WJB-5 strain (harvested from a 30-d-old culture grown on PDA at $20^{\circ} \mathrm{C}$ in the dark) were suspended in sterile distilled water to the final concentration of $3 \times 10^{7}$ conidia $/ \mathrm{mL}$. The resulting conidial suspension was poured on PDA plates and incubated at $20^{\circ} \mathrm{C}$ in darkness, and used for culturing of singleconidium isolates. A total of 100 single-conidium isolates derived from WJB-5 were obtained and separately incubated on PDA plates at $20^{\circ} \mathrm{C}$ for mycelial growth. After the colonies had grown, they were subjected to dsRNA extraction to detect the described virus infection.

\section{Growth Rate and Morphological Assessments}

Morphological traits of virus-infected strains (WJT-1-1, WJT1-3, WJT-1-7, WJT-1-9, WJT-1-12, and WJT-1-13) and viruseliminated strains (WJB-5, WJB-1-1, WJB-1-2, WJB-1-9, WJB1-12, and WJB-1-20) of $M$. theae were assessed as previously described (Jia et al., 2017). Briefly, freshly grown mycelial discs (5 $\mathrm{mm}$ in diameter) were transferred onto PDA and incubated in darkness at $25^{\circ} \mathrm{C}$. Colony diameter was measured daily for 9 days to calculate growth rates $(\mathrm{mm} / \mathrm{d})$.

\section{RESULTS}

\section{Extraction of dsRNA Segments From Melanconiella theae Strains}

To check whether Melanconiella fungi harbor mycoviruses, six strains (WJB-1-1, WJB-1-2, WJB-1-9, WJB-1-12, WJB-120 , and WJB-5) from $M$. theae isolated from Hubei province were randomly chosen from the fungal collection and subjected to dsRNA extraction. It revealed that all strains of the 


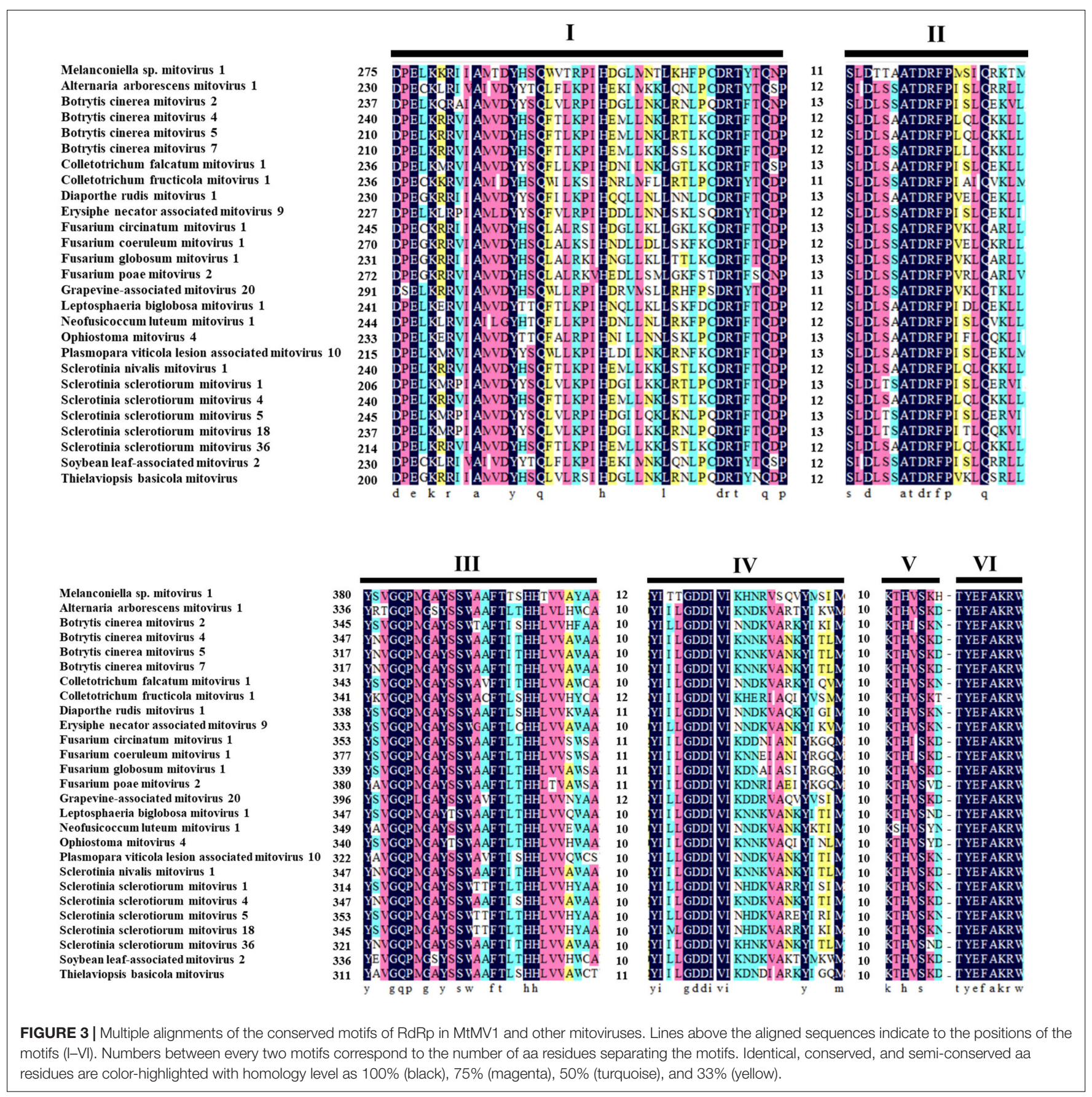

phytopathogenic fungus $M$. theae contain mycovirus-like dsRNA segments (Figure 1). After digestion with DNase I and S1 nuclease enzymes, for the strain WJB-5, a clear band of dsRNA approximately $2.40 \mathrm{~kb}$ was detected (Figure 1).

\section{Sequence and Molecular Characterization of WJB-5 dsRNA}

The entire cDNA sequence of the dsRNA fragment of the strain WJB-5 was obtained through random priming cDNA synthesis, RT-PCR, and RACE cloning. Sequence assembly of thirty random cDNA clones revealed that the genome was composed of a single dsRNA segment with a molecular size of $2,461 \mathrm{bp}$ and with an AU-rich content of 62.37\%. Searching of the full-length genome and the deduced amino acid sequences using BLASTX and BLASTP, the coding protein shared 37.77$48.65 \%$ identities with the RdRp sequences of known mitoviruses belonging to the family Mitoviridae, and the highest identities are 48.65 and $48.35 \%$ with those of grapevine-associated mitovirus 20 (GaMV20) and Colletotrichum fructicola mitovirus 1 (CfMV1), respectively (Supplementary Table 2). Thus, the dsRNA component is proposed as a novel mitovirus and 


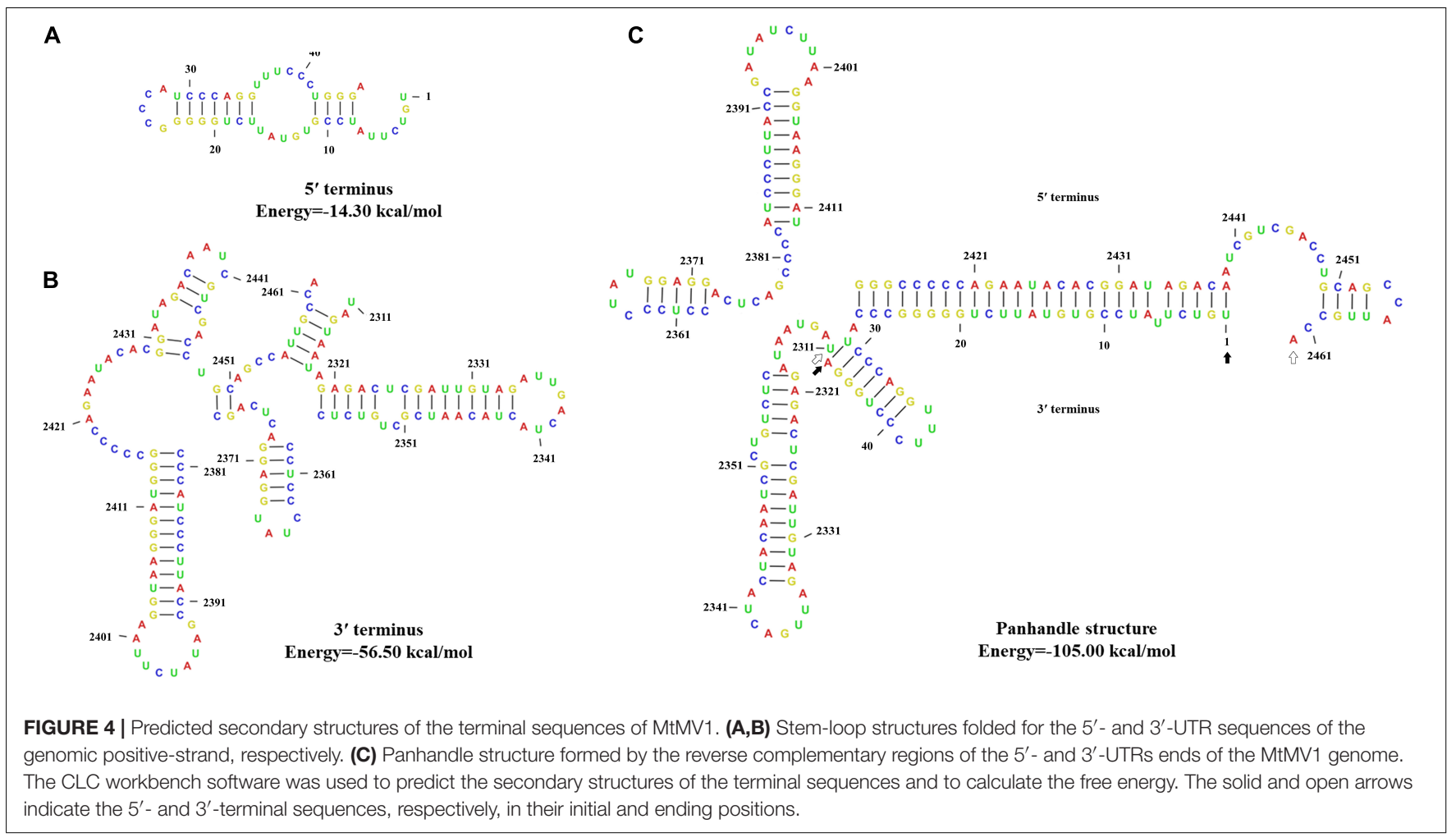

tentatively named Melanconiella theae mitovirus 1 (MtMV1). The complete nucleotide sequence of MtMV1 has been deposited in GenBank with the accession number MW802251.

\section{Genomic Organization and Phylogenetic Analysis}

Based on the yeast mitochondrial code, the genomic organization analysis revealed that the MtMV1 genome contains a single large ORF on the positive sense strand of the dsRNA (Figure 2A).

The ORF starts and terminates at nucleotide positions 46 and 2,310 , respectively, of 2,265 bp in size, and potentially codes for an RdRp protein with 754 aa in size. The ORF contains multiple codons (10 UGA and 2 UGG) encoding Trp, and the UAG codon stops the translation. Moreover, the $5^{\prime}$ - and $3^{\prime}$-untranslated regions (UTRs) are 45 and 151 bp long, respectively (Table 1). The phylogenetic analysis separated the known mitoviruses into two distinct clusters and placed MtMV1 together with CfMV1 and GaMV20 in cluster I, which is distantly separated from other members (Figure 2B). Based on the conserved domain database (CDD) search and multiple amino acid alignments, the six typical amino acid motifs (I-IV) characterized for the mitoviral RdRp domain superfamily were found to be conserved in MtMV1 (Figure 3). However, MtMV1 contains many unique amino acids as compared with the other members in the conserved motifs (e.g., positions 12 and 20 in motif I, 4, and 19 in motif II, 18 in motif III, 3 and 4 in motif IV), which were composed by threonines (Thr) for MtMV1 while leucines (Leu), isoleucines (Ile) or valines (Val) for the others (Figure 3). Moreover, an unique serine (Ser) in position 16 in motif IV and a unique histidine (His) in position 7 in motif $\mathrm{V}$ were observed for MtMV1, while alanine in motif IV and aspartic acids, asparagines or threonines in motif $\mathrm{V}$ in these positions for the others (Figure 3).

\section{Predicted Secondary Structures of the Terminal Sequence}

The $5^{\prime}$ - and $3^{\prime}$-terminus sequences of the MtMV1 genome were subjected to the prediction of their secondary structures using the CLC genomic workbench software. The results showed that both $5^{\prime}$ - and $3^{\prime}$-UTRs could be folded into potential stem-loop structures that stabilize the RNA genome, with $\Delta G$ values of -14.3 and $-56.5 \mathrm{kcal} / \mathrm{mol}$, respectively (Figures 4A,B). Additionally, the partial regions of both terminal sequences are reverse complementary, with positions 1 to 27 of $5^{\prime}$-UTR paired with positions 100 to 126 of $3^{\prime}$-UTR, and potentially fold into a panhandle structure with $\Delta \mathrm{G}$ values of $-105 \mathrm{kcal} / \mathrm{mol}$ (Figure 4C).

\section{Attempts to Check the Viral-Like Particles From WJB-5 Strain}

To determine whether virions are associated with MtMV1, possible viral proteins were extracted from the mycelia of strain WJB-5 and subjected to ultracentrifugation in stepwise sucrose gradients. The sucrose fraction containing the MtMV1 dsRNAs was further purified and subjected to observation with a transmission electron microscope (TEM). No virus-like particles were observed under TEM, confirming that MtMV1 dsRNAs are naked like other mitoviruses. 
A

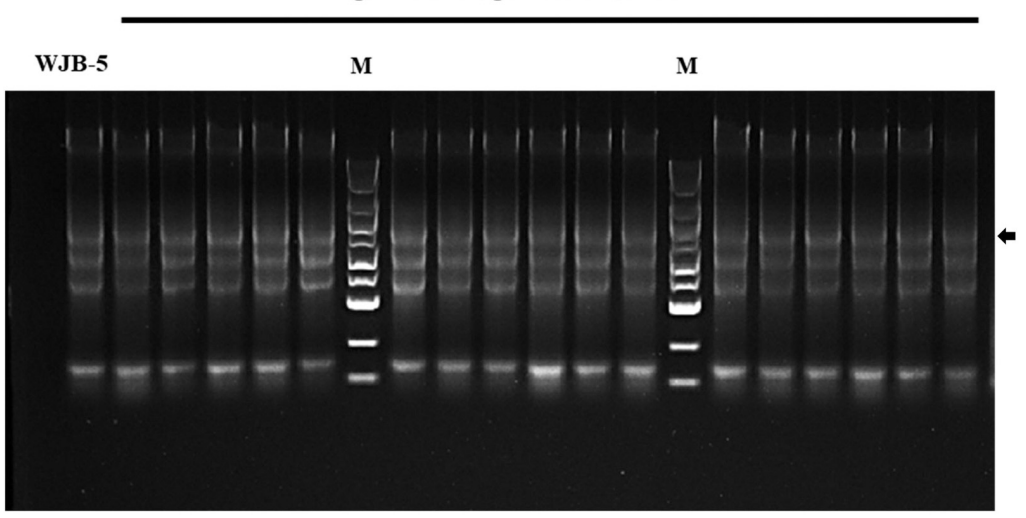

B

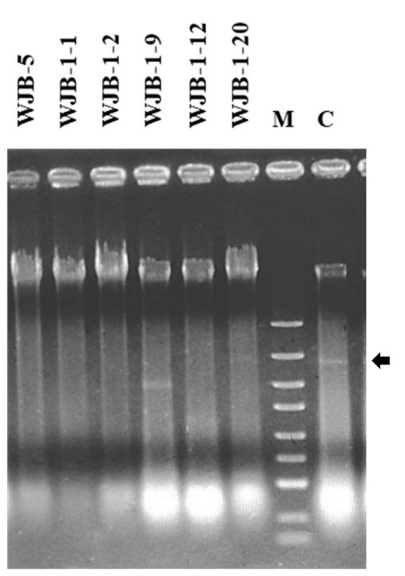

FIGURE 5 | Agarose gel electrophoresis of dsRNA extracted from the mycelia of Melanconiella theae strains. (A) dsRNA extraction from the mycelia of single-conidium generated colonies derived from strain WJB-5 conidia. (B) dsRNA extraction from six strains (WJB-5, WJB-1-1, WJB-1-2, WJB-1-9, WJB-1-12, and WJB-1-20) after being treated with hyphal tipping combined with antiviral extracts. (C) Untreated WJB-5 as a control. M: DL5000 DNA marker (100; 250; 500; 750; 1,000; 1,500; 2,000; 3,000; and 5,000 bp). The arrows indicate the target viral dsRNA bands.

\section{Attempts to Cure WJB-5 Strain From Melanconiella Theae Mitovirus 1}

To cure the MtMV1 from the WJB-5 strain and the other five strains of $M$. theae (WJB-1-1, WJB-1-2, WJB-1-9, WJB-1-12, and WJB-1-20), the hyphal tipping method combined with antiviral extracts (incorporating green-leaf tea powder in the culture medium) was used. After three sequential rounds of subculturing, MtMV1 was detected by dsRNA extraction. The results showed that MtMV1 was cured from all detected strains (Figure 5B), which was further confirmed by RT-PCR identification resulting in no target bands (1.98 kb in size) (Supplementary Figure 2).

\section{Vertical Transmission of Melanconiella Theae Mitovirus 1 Through Conidia}

To investigate whether MtMV1 can be vertically transmitted to the next generation through fungal conidia, conidial suspension prepared from the strain WJB-5 were smeared on PDA, and a total of 100 single-conidium generated colonies were subjected to dsRNA extraction. The results showed that MtMV1 was retained in all these subisolates, suggesting that MtMV1 has efficiently transmitted through the conidia of $M$. theae (Figure 5A).

\section{Melanconiella theae Mitovirus 1 Confers No Obvious Effects on the Growth Rates and Morphologies of the Host Fungus}

To access the effect of MtMV1 on the host fungus, six MtMV1infected and six MtMV1-free strains of Melanconiella spp. were cultured on PDA in darkness to access their growth rates and morphologies. Most of these strains had similar morphologies and growth rates, ranging from 4.4 to $6.1 \mathrm{~mm} / \mathrm{d}$ for the MtMV1infected strains and 4.1 to $5.4 \mathrm{~mm} / \mathrm{d}$ for the MtMV1-free ones, suggesting that no obvious effects were observed for MtMV1 related to the morphologies and growth rates of their host strains (Supplementary Figure 3).

\section{DISCUSSION}

A novel mycovirus, named MtMV1, was identified infecting the phytopathogenic fungus $M$. theae. MtMV1 shares most characteristics of members in the family Mitoviridae considering that mitoviruses have the simplest positive ssRNA genomes that harbor a single ORF encoding a putative viral RdRp for their replication (Cole et al., 2000; Hillman and Cai, 2013; Khalifa and Pearson, 2013), capsidless (Hillman and Cai, 2013), and their RdRps contain six conserved amino acid motifs (I-IV) (Osaki et al., 2015; Ran et al., 2016; Chen et al., 2017; Wang et al., 2019; Kocanová et al., 2020; Liu et al., 2021). Consequently, MtMV1 together with GaMV20 and CFMV1 were placed in cluster I as the third member in this group.

Since mitoviruses essentially replicate within mitochondria of their hosts (Polashock and Hillman, 1994; Cole et al., 2000; Osaki et al., 2005; Nibert, 2017; Fonseca et al., 2021), MtMV1 genome shares the most features of mitochondrial codons described before, e.g., the MtMV1 genome contains ten UGA codons, which encode Trp rather than function as a stop codon (Nibert, 2017; Vong et al., 2019), while only two UGG, and has rich of A-U content (62.37\%) like other members (59.55 to $73.25 \%$ ). Moreover, the initiation codon of MtMV1 ORF has a preference to be either $\mathrm{A}$ or $\mathrm{U}$ with a frequency of $68.3 \%$ in the third nucleotide position, similar to those of other members (Liu et al., 2019; Torres-Trenas and Pérez-Artés, 2020; Wang et al., 2021) and has a UAG stop codon, similar to the observation in other members with either UAA or UAG (Nibert, 2017). Additionally, the $5^{\prime}$ - and $3^{\prime}$-terminus sequences of MtMV1 could be folded into stem-loop structures, and their reverse complementary regions allow to form a stable panhandle structure, which is also observed in other members (Hong et al., 1999; Khalifa and Pearson, 2013; Wang et al., 2021). Whereas, the size of MtMV1 3'-UTR (151 $\mathrm{nt}$ ) is relatively longer than its $5^{\prime}$-UTR (45 nt), it is different as compared with other mitoviruses since their $3^{\prime}$-UTR sizes are all shorter than their $5^{\prime}$-UTR (ranging from 1 to 413 nucleotide 
longer) (Stielow et al., 2011; Xie and Ghabrial, 2012; Nibert et al., 2018). It is worth noting that MtMV1 has the smallest $5^{\prime}$-UTR in size compared with other members (ranging from 49 to $472 \mathrm{nt}$ ) in the family Mitoviridae whether some biological functions related to the unique traits of MtMV1 terminal sequences require further studies. Moreover, MtMV1 harbors a genomic sequence obviously divergent from those of most members in the family Mitoviridae as exemplified by having Thr-rich RdRp motifs as compared with other members (Figure 3).

MtMV1 is efficiently transmitted through all conidia of strain WJB-5 (100 conidium-generated colonies were checked) in agreement with those mitoviruses detected in a high percentage of the individual spore isolates, as exemplified by Fusarium circinatum mitovirus 1 (FcMV1) and Fusarium circinatum mitovirus 2-2 (FcMV2-2) that showed vertical transmission rates between 60 and 100\% depending on the fungal isolate (Romeralo et al., 2018), however, both Fusarium verticillioides mitovirus 1 (FvMV1) and Fusarium andiyazi mitovirus 1 strain 162 (FaMV1162) showed $100 \%$ vertical transmission rate through fungal conidia (Jacquat et al., 2020). In contrast, some mycoviruses are less efficiently transmitted through conidia, as exemplified by Colletotrichum camelliae filamentous virus 1 (CcFV-1) and Pestalotiopsis theae chrysovirus-1 (PtCV1) (Jia et al., 2017; Zhou et al., 2021). Generally, the efficient transmission of mitoviruses through asexual spores and sclerotia has hindered to understand mitovirus biology, partly due to lacking mitovirus-free subisolates used for infection, although a protoplast fusion-based protocol for horizontal transmission of a mitovirus has been developed (Shahi et al., 2019). In light of several reports of tea polyphenols exhibiting antiviral activities against various viruses, especially positive-sense single-stranded RNA viruses (Okada, 1978; Zhang et al., 2020; Mhatre et al., 2021), we tried the elimination of MtMV1 from the fungal host, it is worth noting that MtMV1 was easily cured by the hyphal tipping method combined with green-leaf tea powder in the culture medium as examined by dsRNA extraction and RT-PCR identification, which is expected to provide an efficient approach to obtain mitovirus-free host strains. Moreover, MtMV1 confers no obvious effects on the growth rates and morphologies as demonstrated by some homologous and isogenous strains. Thus, we did not proceed to viral transmission for more strict assessment of its biological effects.

Collectively, a novel mitovirus (MtMV1) from $M$. theae was characterized based on its taxonomic analysis, molecular characterization, genome organization, electron microscope

\section{REFERENCES}

Abid, M., Khan, M. A. U., Mushtaq, S., Afzaal, S., and Haider, M. S. (2018). A Comprehensive Review on Mycoviruses as Biological Control Agent. World J. Biol. Biotechnol. 3, 187-192. doi: 10.33865/wjb.003.02. 0146

Chen, Y., Shang, H. H., Yang, H. Q., da Gao, B., and Zhong, J. (2017). A mitovirus isolated from the phytopathogenic fungus Alternaria brassicicola. Arch. Virol. 162, 2869-2874. doi: 10.1007/s00705-017-3371-8

Cole, T. E., Hong, Y., Brasier, C. M., and Buck, K. W. (2000). Detection of an RNA-dependent RNA polymerase in mitochondria from a mitovirus-infected observation, and some biological traits. MtMV1 harbors a genomic sequence obviously divergent with those of most members in the family Mitoviridae and some unique characteristics unreported in known members, contributing helpful information to understand the evolution, molecular and biological traits of mycoviruses.

\section{DATA AVAILABILITY STATEMENT}

The datasets presented in this study can be found in online repositories. The names of the repository/repositories and accession number(s) can be found in the article/ Supplementary Material.

\section{AUTHOR CONTRIBUTIONS}

WX supervised and designed the experiments. KS conducted most of the experiments and wrote the manuscript. MU improved the English and conducted part of experiments. KS and MU analyzed the data. HY and HA conducted initial experiments. MU, HA, ZW, DN, and $\mathrm{WX}$ revised the manuscript. All authors read and agreed to the published version of the manuscript.

\section{FUNDING}

This research was funded by the National Natural Science Foundation of China, grant numbers 31872014 and 32172475 to WX.

\section{ACKNOWLEDGMENTS}

The authors thank the financial support by the National Natural Science Foundation of China and Hubei Hongshan Laboratory.

\section{SUPPLEMENTARY MATERIAL}

The Supplementary Material for this article can be found online at: https://www.frontiersin.org/articles/10.3389/fmicb. 2021.757556/full\#supplementary-material

isolate of the dutch elm disease fungus. Ophiostoma novo- ulmi. Virology 268, 239-243. doi: 10.1006/viro.1999.0097

Du, Z., Fan, X. L., Yang, Q., and Tian, C. M. (2017). Host and geographic range extensions of Melanconiella, with a new species M. Cornuta in China. Phytotaxa 327, 252-260. doi: 10.11646/phytotaxa.327.3.4

Fonseca, P., Ferreira, F., da Silva, F., Oliveira, L. S., Marques, J. T., Goes-Neto, A., et al. (2021). Characterization of a novel mitovirus of the sand fly Lutzomyia longipalpis using genomic and virus-host interaction signatures. Viruses 13:9. doi: 10.3390/v13010009

Ghabrial, S. A. (1998). Origin, adaptation and evolutionary pathways of fungal viruses. Virus Genes 16, 119-131. doi: 10.1023/a:1007966229595 
Ghabrial, S. A., and Suzuki, N. (2009). Viruses of plant pathogenic fungi. Аnnu. Rev. Phytopathol. 47, 353-384. doi: 10.1146/annurev-phyto-080508-081932

He, H., Chen, X., Li, P., Qiu, D., and Guo, L. (2018). Complete genome sequence of a Fusarium graminearum double-stranded RNA virus in a newly proposed family, Alternaviridae. Genome Announc. 6, e00064-18. doi: 10.1128/genomeA. 00064- 18

Hillman, B. I., and Cai, G. (2013). The family Narnaviridae: simplest of RNA viruses. Adv. Virus Res. 86, 149-176. doi: 10.1016/B978-0-12-394315-6.00 006-4

Hong, Y., Cole, T. E., Brasier, C. M., and Buck, K. W. (1998). Evolutionary relationships among putative RNA-dependent RNA polymerases encoded by a mitochondrial virus-like RNA in the dutch elm disease fungus, Ophiostoma novo-ulmi, by other viruses and virus-like RNAs and by the Arabidopsis mitochondrial genome. Virology 246, 158-169. doi: 10.1006/viro.1998.9178

Hong, Y., Dover, S. L., Cole, T. E., Brasier, C. M., and Buck, K. W. (1999). Multiple mitochondrial viruses in an isolate of the dutch elm disease fungus Ophiostoma novo-ulmi. Virology 258, 118-127. doi: 10.1006/viro.1999.9691

Jacquat, A. G., Theumer, M. G., Cañizares, M. C., Debat, H. J., Iglesias, J., Pedrajas, M. D. G., et al. (2020). A survey of mycoviral infection in Fusarium spp. isolated from maize and sorghum in argentina identifies the first mycovirus from Fusarium verticillioides. Viruses 12:1161. doi: 10.3390/v12101161

Jia, H., Dong, K., Zhou, L., Wang, G., Hong, N., Jiang, D., et al. (2017). A dsRNA virus with filamentous viral particles. Nat. Commun. 8:168. doi: 10.1038/ s41467-017-00237-9

Kamaruzzaman, M., He, G., Wu, M., Zhang, J., Yang, L., Chen, W., et al. (2019). A novel partitivirus in the hypovirulent isolate qt5-19 of the plant pathogenic fungus Botrytis cinerea. Viruses 11:24. doi: 10.3390/v11010024

Khalifa, M. E., and Pearson, M. N. (2013). Molecular characterization of three mitoviruses co-infecting a hypovirulent isolate of Sclerotinia sclerotiorum fungus. Virology 441, 22-30. doi: 10.1016/j.virol.2013.03.002

Kocanová, M., Eichmeier, A., and Botella, L. (2020). A novel mitovirus detected in Diaporthe rudis, a fungus associated with Phomopsis dieback on grapevines. Arch. Virol. 165, 2405-2408. doi: 10.1007/s00705-020-04755-8

Lim, W. S., Jeong, J. H., Jeong, R. D., Yoo, Y. B., Yie, S. W., and Kim, K. H. (2005). Complete nucleotide sequence and genome organization of a dsRNA partitivirus infecting Pleurotus ostreatus. Virus Res. 108, 111-119. doi: 10.1016/ j.virusres.2004.08.017

Liu, C. H., and Di, Y. P. (2020). "Analysis of RNA sequencing data using CLC genomics workbench," in Methods in Molecular Biology, eds P. Keohavong, K. P. Singh, and W. Gao (New York: Springer), 61-113. doi: 10.1007/978-1-07160223-2_4

Liu, H., Liu, M., Zhu, H., Zhong, J., Liao, X., and Zhou, Q. (2021). Molecular characterization of a novel mitovirus from the plant-pathogenic fungus Botryosphaeria dothidea. Arch. Virol. 166, 633-637. doi: 10.1007/s00705-02004886-y

Liu, H., Liu, R., Li, C. X., Wang, H., Zhu, H. J., da Gao, B., et al. (2019). A victorivirus and two novel mitoviruses co-infected the plant pathogen Nigrospora oryzae. Viruses 11:83. doi: 10.3390/v11010083

Liu, L., Xie, J., Cheng, J., Fu, Y., Li, G., Yi, X., et al. (2014). Fungal negative-stranded RNA virus that is related to bornaviruses and nyaviruses. Proc. Natl. Acad. Sci. U. S. A. 111, 12205-12210. doi: 10.1073/pnas.1401786111

Magae, Y. (2012). Molecular characterization of a novel mycovirus in the cultivated mushroom, Lentinula edodes. Virol. J. 9:60. doi: 10.1186/1743-422X-9-60

Mhatre, S. S. T., Naik, S., and Patravale, V. (2021). Antiviral activity of green tea and black tea polyphenols in prophylaxis and treatment of COVID-19: a review. Phytomedicine 85:153286. doi: 10.1016/j.phymed.2020.153286

Mizutani, Y., Abraham, A., Uesaka, K., Kondo, H., Suga, H., Suzuki, N., et al. (2018). Novel mitoviruses and a unique tymo-like virus in hypovirulent and virulent strains of the Fusarium head blight fungus, Fusarium boothii. Viruses 10:584. doi: 10.3390/v10110584

Nerva, L., Vigani, G., di Silvestre, D., Ciuffo, M., Forgia, M., Chitarra, W., et al. (2019). Biological and molecular characterization of Chenopodium quinoa mitovirus 1 reveals a distinct small RNA response compared to those of cytoplasmic RNA viruses. J. Virol. 93, e01998-18. doi: 10.1128/jvi.01998-18

Nibert, M. L. (2017). Mitovirus UGA(Trp) codon usage parallels that of host mitochondria. Virology 507, 96-100. doi: 10.1016/j.virol.2017.04.010
Nibert, M. L., Debat, H. J., Manny, A. R., Grigoriev, I. V., and de Fine Licht, H. H. (2019). Mitovirus and mitochondrial coding sequences from basal fungus Entomophthora muscae. Viruses 11:351. doi: 10.3390/v11040351

Nibert, M. L., Vong, M., Fugate, K. K., and Debat, H. J. (2018). Evidence for contemporary plant mitoviruses. Virology 518, 14-24. doi: 10.1016/j.virol.2018. 02.005

Nuss, D. L. (2005). Hypovirulence: mycoviruses at the fungal-plant interface. Nat. Rev. Microbiol. 3, 632-642. doi: 10.1038/nrmicro1206

Okada, F. (1978). Antiviral effects of tea catechins and black tea theaflavins on plant viruses. JARQ 12, 27-32.

Osaki, H., Nakamura, H., Nomura, K., Matsumoto, N., and Yoshida, K. (2005). Nucleotide sequence of a mitochondrial RNA virus from the plant pathogenic fungus, Helicobasidium mompa Tanaka. Virus Res. 107, 39-46. doi: 10.1016/j. virusres.2004.06.010

Osaki, H., Sasaki, A., Nomiyama, K., Sekiguchi, H., Tomioka, K., and Takehara, T. (2015). Isolation and characterization of two mitoviruses and a putative alphapartitivirus from Fusarium spp. Virus Genes 50, 466-473. doi: 10.1007/ s11262-015-1182-0

Pearson, M. N., Beever, R. E., Boine, B., and Arthur, K. (2009). Mycoviruses of filamentous fungi and their relevance to plant pathology. Mol. Plant Pathol. 10, 115-128. doi: 10.1111/j.1364-3703.2008.00503.x

Polashock, J. J., and Hillman, B. I. (1994). A small mitochondrial double-stranded (ds) RNA element associated with a hypovirulent strain of the chestnut blight fungus and ancestrally related to yeast cytoplasmic T and W dsRNAs. Proc. Natl. Acad. Sci. U. S. A. 91, 8680-8684. doi: 10.1073/pnas.91.18.8680

Ran, H., Liu, L., Li, B., Jiasen, C., Fu, Y., Daohong, J., et al. (2016). Co-infection of a hypovirulent isolate of Sclerotinia sclerotiorum with a new botybirnavirus and a strain of a mitovirus. Virol. J. 13:92. doi: 10.1186/s12985-016-0550-2

Ro, H. S., Kang, E. J., Yu, J. S., Lee, T. S., Lee, C. W., and Lee, H. S. (2007). Isolation and characterization of a novel mycovirus, PeSV, in Pleurotus eryngii and the development of a diagnostic system for it. Biotechnol. Lett. 29, 129-135. doi: 10.1007/s10529-006-9206-4

Romeralo, C., Bezos, D., Martínez-álvarez, P., and Diez, J. J. (2018). Vertical transmission of Fusarium circinatum mitoviruses FcMV1 and FcMV2-2 via microconidia. Forests 9:356. doi: 10.3390/f9060356

Sahin, E., and Akata, I. (2019). Complete genome sequence of a novel mitovirus from the ectomycorrhizal fungus Geopora sumneriana. Arch. Virol. 164, 28532857. doi: 10.1007/s00705-019-04367-x

Shahi, S., Eusebio-Cope, A., Kondo, Hideki B, Hillman, I., and Suzuki, N. (2019). Investigation of host range of and host defense against a mitochondrially replicating mitovirus. J. Virol. 93, e1503-e1518.

Stielow, B., Klenk, H. P., Winter, S., and Menzel, W. (2011). A novel Tuber aestivum (Vittad.) mitovirus. Arch. Virol. 156, 1107-1110. doi: 10.1007/s00705011-0998-8

Strauss, E. E., Lakshman, D. K., and Tavantzis, S. M. (2000). Molecular characterization of the genome of a partitivirus from the basidiomycete Rhizoctonia solani. J. Gen. Virol. 81, 549-555.

Toni, L. S., Garcia, A. M., Jeffrey, D. A., Jiang, X., Stauffer, B. L., Miyamoto, S. D., et al. (2018). Optimization of phenol-chloroform RNA extraction. MethodsX 5, 599-608. doi: 10.1016/j.mex.2018.05.011

Torres-Trenas, A., and Pérez-Artés, E. (2020). Characterization and incidence of the first member of the genus Mitovirus identified in the phytopathogenic species Fusarium oxysporum. Viruses 12:279. doi: 10.3390/v12030279

Voglmayr, H., Rossman, A. Y., Castlebury, L. A., and Jaklitsch, W. M. (2012). Multigene phylogeny and taxonomy of the genus Melanconiella (Diaporthales). Fungal Divers. 57, 1-44. doi: 10.1007/s13225-012-0175-8

Vong, M., Manny, A. R., Smith, K. L., Gao, W., and Nibert, M. L. (2019). Beta vulgaris mitovirus 1 in diverse cultivars of beet and chard. Virus Res. 265, 80-87. doi: 10.1016/j.virusres.2019.02.008

Wang, H., Liu, H., and Zhou, Q. (2021). The complete genome sequence of a new mitovirus from the phytopathogenic fungus Colletotrichum higginsianum. Arch. Virol. 166, 1481-1484. doi: 10.1007/s00705-021-04996-1

Wang, Y., Xu, Z., Cheng, J., Fu, Y., Jiang, D., and Xie, J. (2019). Characterization of a novel RNA virus from the phytopathogenic fungus Leptosphaeria biglobosa related to members of the genus Mitovirus. Arch. Virol. 164, 913-916. doi: 10.1007/s00705-018-04143-3 
Wu, M. D., Zhang, L., Li, G. Q., Jiang, D. H., Hou, M. S., and Huang, H. C. (2007). Hypovirulence and double-stranded RNA in Botrytis cinerea. Phytopathology 97, 1590-1599. doi: 10.1094/PHYTO-97-12-1590

Xie, J., and Ghabrial, S. A. (2012). Molecular characterizations of two mitoviruses co-infecting a hyovirulent isolate of the plant pathogenic fungus Sclerotinia sclerotiorum. Virology 428, 77-85. doi: 10.1016/j.virol.2012.03.015

Xie, J., Xiao, X., Fu, Y., Liu, H., Cheng, J., Ghabrial, S. A., et al. (2011). A novel mycovirus closely related to hypoviruses that infects the plant pathogenic fungus Sclerotinia sclerotiorum. Virology 418, 49-56. doi: 10.1016/j.virol.2011. 07.008

Xu, Z., Wu, S., Liu, L., Cheng, J., Fu, Y., Jiang, D., et al. (2015). A mitovirus related to plant mitochondrial gene confers hypovirulence on the phytopathogenic fungus Sclerotinia sclerotiorum. Virus Res. 197, 127-136. doi: 10.1016/j.virusres. 2014.12.023

Yang, D., Wu, M., Zhang, J., Chen, W., Li, G., and Yang, L. (2018). Sclerotinia minor endornavirus 1, a novel pathogenicity debilitation-associated mycovirus with a wide spectrum of horizontal transmissibility. Viruses 10:589. doi: 10.3390/ v10110589

Yang, F., Wang, G., Xu, W., and Hong, N. (2017). A rapid silica spin columnbased method of RNA extraction from fruit trees for RT-PCR detection of viruses. J. Virol. Methods 247, 61-67. doi: 10.1016/j.jviromet.2017.0 5.020

Yang, Y., and Zhang, T. (2019). Antimicrobial activities of tea polyphenol on phytopathogens: a review. Molecules 24:816. doi: 10.3390/molecules2404 0816

Yu, X., Li, B., Fu, Y., Jiang, D., Ghabrial, S. A., Li, G., et al. (2010). A geminivirusrelated DNA mycovirus that confers hypovirulence to a plant pathogenic fungus. Proc. Natl. Acad. Sci. U. S. A. 107, 8387-8392. doi: 10.1073/pnas. 0913535107

Zhang, X. N., Liao, Y. W., Wang, X. R., Zhang, L., Ahammed, G. J., Li, Q. Y., et al. (2020). Epigallocatechin-3-gallate enhances tomato resistance to tobacco mosaic virus by modulating RBOH1-dependent $\mathrm{H} 2 \mathrm{O} 2$ signaling. Plant Physiol. Biochem. 150, 263-269. doi: 10.1016/j.plaphy.2020.03.008

Zhou, L., Li, X., Kotta-Loizou, I., Dong, K., Li, S., Ni, D., et al. (2021). A mycovirus modulates the endophytic and pathogenic traits of a plant associated fungus. ISME J. 15, 1893-1906. doi: 10.1038/s41396-021-00892-3

Conflict of Interest: The authors declare that the research was conducted in the absence of any commercial or financial relationships that could be construed as a potential conflict of interest.

Publisher's Note: All claims expressed in this article are solely those of the authors and do not necessarily represent those of their affiliated organizations, or those of the publisher, the editors and the reviewers. Any product that may be evaluated in this article, or claim that may be made by its manufacturer, is not guaranteed or endorsed by the publisher.

Copyright (c) 2021 Shafik, Umer, You, Aboushedida, Wang, Ni and Xu. This is an open-access article distributed under the terms of the Creative Commons Attribution License (CC BY). The use, distribution or reproduction in other forums is permitted, provided the original author(s) and the copyright owner(s) are credited and that the original publication in this journal is cited, in accordance with accepted academic practice. No use, distribution or reproduction is permitted which does not comply with these terms. 